\title{
IF YOU ARE SO RICH, WHY AREN'T YOU SMART?
}

\author{
Nobuyuki Hanaki \\ Juliette Rouchier \\ Aix-Marseille University (Aix-Marseille School of Economics), CNRS \& EHESS \\ 2 Rue de la Charité. 13002 Marseille, FRANCE.
}

\begin{abstract}
We consider a differentiated-goods Cournot competition where each agent learns about how much to produce. There are two types of agents: ignorant and informed. Ignorants do not know about the demand function for their products and naively assume prices for their products will remain the same as the previous period in the process of learning. Informed ones, on the other hand, know about the demand function, and learn how much to produce by myopically best responding against the quantities produced by others. We show that there are situations in which ignorants are more successful than informed (in a sense that they obtain a higher payoff than the latter). This occurs because of the way two types of agents learn to behave. Of course, there are situations where the opposite, the informed being richer than the ignorants, is true. The nature of strategic interactions determines which outcome prevails.
\end{abstract}

\section{INTRODUCTION}

Can less smart people become richer than smarter ones not by luck but due to the way they behave? We address this question by considering very simple worlds in which there are two types of adaptive agents: ignorant and informed. Agents of the former type do not know about the environment they face and learn what to do in a naive way, while agents of the latter type know quite a bit about the environment and learn what to do in a more sophisticated manner. We assume that none of the agents knows explicitly how others make their decisions (or learn). In this simple set up, we can say that informed ones are smarter than ignorants, although even informed ones are quite naive as they are not aware of (nor learn about) the heterogeneity among agents.

In particular, we consider a differentiated-goods Cournot competition where each agent learns how much to produce. An ignorant does not know about the demand function for his product and naively assumes the price of his product will remain the same as the previous period in the process of learning. An informed agent, on the other hand, knows about the demand function, and learns how much to produce by myopically best responding against the total quantity produced by others. That is, the informed agent considers the effect of his changing his production level on the price of his product in the process of learning, although the informed agent naively assumes that the others will keep their outputs fixed at the previous level.

We have chosen a Cournot competition because learning in this setting has been studied extensively both theoretically (including agent-simulation) and experimentally. The reason for this interest lies in the convergence properties of different learning models. For example, it has been shown that, under a homogeneous-good Cournot competition, while the best reply dynamics converge to Cournot-Nash equilibrium, an imitation-based learning (social learning) converges to Walrasian equilibrium (Vega-Redondo 1997, Vriend 2000, Vallée and Yildizoğlu 2009). This prediction has been tested experimentally and found comparative statics supports as well. See, for example, Huck, Normann, and Oechssler (1999), Huck, Normann, and Oechssler (2000), Offerman, Potters, and Sonnemans (2002), Apesteguia, Huck, and Oechssler 


\section{Hanaki and Rouchier}

(2007). What have not been investigated much are situations where agents are following heterogeneous adaptive rules. What we consider in this paper is one of such situations.

What we show in this paper is that there are situations where ignorants are more successful than informed agents (in a sense that they obtain a higher payoff than the latter). Thus the answer to the above question can be positive. In fact, in our simple world, when ignorants are more successful than informed, it is the behaviors of informed that allow the ignorants to outperform them. Of course, there are situation where the opposite, the informed being more successful than the ignorants, is true. We show that the nature of the strategic interaction among agents plays a crucial role in determining which outcome will prevail. Haltiwanger and Waldman (1985) and Haltiwanger and Waldman (1989) have shown the importance of the nature of strategic interaction, in particular, whether there exists strategic complementarity (positive feedback) or strategic substitution (negative feedback), in determining aggregate outcomes when agents are heterogenous in their depth of strategic thinking. This theoretical finding has been confirmed experimentally in such games as a price setting game (Fehr and Tyran 2008), price-forecasting games (Heemeijer et al. 2009, Bao et al. 2012), and beauty contest games (Sutan and Willinger 2009). Our choice of differentiated goods, instead of a homogeneous good, Cournot competition allows us to consider both the strategic substitutions and complementarities just by changing the value of one parameter.

In our model of differentiated goods markets, when the goods produced by agents are close substitutes (so that there exists strong strategic substitutions), then ignorants learn to produce the quantities that result in higher profits than informed agents. In fact, in such cases, while ignorants are, on average, learning to produce the quantity that equates the marginal cost and the price (thus producing more than the CournotNash equilibrium level), informed agents learn to respond by cutting back their productions (thus, informed agents produce less than the Cournot-Nash equilibrium level). This response by informed agents to cut back their productions is what makes ignorants richer than the informed agents. On the other hand, when goods are complements (so that there exists strategic complementarities), then, informed agents will learn to obtain higher profits than the ignorants. As in the case where goods are close substitutes, ignorants will learn to increase their productions up to the point where marginal cost is equal to the price. This increase in their productions, however, is detrimental to their profits (because it lowers the price of own good) while helps to increase the profits of others (by raising their prices) when goods are complements. When the degree of substitution among goods is low (thus the degree of strategic substitution is also low), an ignorant increasing his production reduces the profits of all the agents. But the negative effect to the profit is the largest for the ignorant increasing the production than for the others.

The rest of the paper is organized as follows: Section 2 introduces our model. Results of analyses, both computational experiments and mathematical ones, are summarized and discussed in Section 3. Section 4 concludes.

\section{MODEL}

\subsection{Market}

We consider a world with $\mathrm{N}$ agents that produce differentiated goods. Agent $i$ is facing a market characterized by an inverse demand function such that

$$
P_{i}\left(q_{i}, Q_{-i}\right)=a-b q_{i}-c Q_{-i}
$$

where $q_{i}$ in is the quantity produced by $i, Q_{-i}=\sum_{j \neq i} q_{j}$ is the total production of agents other than $i$. We assume $|c| \leq|b|, 0<b$, and $0 \ll a$. One can easily see that when $b=c$, we are back to the homogeneous good oligopoly model. We assume $c \neq 0$ for an obvious reason, that is when $c=0$, each agent is a monopolist and thus there is no interaction among agents. When $c>0(c<0)$, goods are substitutes (complements).

We assume a quadratic cost function as in Vallée and Yildizoğlu (2009) but without a fixed cost, i.e.,

$$
C\left(q_{i}\right)=d q_{i}+e q_{i}^{2}
$$




\section{Hanaki and Rouchier}

with conditions $d \geq 0, e>0$ and $d \ll a$. We will further assume that $2 e+b+c(N-1)>0$ to obtain non-negative quantities in symmetric Cournot-Nash and Walrasian equilibria.

With the inverse demand function (1) and the cost function (2), it is straight forward to verify that the symmetric Cournot-Nash equilibrium quantity $\left(q^{C}\right)$ and price $\left(p^{C}\right)$ are

$$
\begin{gathered}
q^{C}(a, b, c, d, e, N)=\frac{a-d}{2(b+e)+c(N-1)} \\
p^{C}(a, b, c, d, e, N)=a-(b+c(N-1)) \frac{a-d}{2(b+e)+c(N-1)}
\end{gathered}
$$

and the quantity and the price at the symmetric Walrasian equilibrium where all the agents produces at $q^{W}$ such that its marginal cost is equal to the price $\left(M C\left(q^{W}\right)=p^{W}\right)$ are

$$
\begin{gathered}
q^{W}(a, b, c, d, e, N)=\frac{a-d}{2 e+b+c(N-1)} \\
p^{W}(a, b, c, d, e, N)=a-(b+c(N-1)) \frac{a-d}{2 e+b+c(N-1)}
\end{gathered}
$$

\subsection{Adaptive Agents}

We consider adaptive agents. That is, each agent learns how much to produce based on the outcomes in the past. As noted in the introduction, we consider two types of agents: Ignorant and Informed. Ignorant agents do not know about the demand function for their products and naively assume prices for their products will remain the same as previous period whenever they learn about how much to produce. Informed agents, on the other hand, know about the demand function they face, and learn how much to produce by myopically best responding against the total quantity produced by others in the previous period (thus, informed do not need to know the quantity supplied by each agent), that is, they consider the changes in prices of their products when they change their quantities assuming that all the others will keep their outputs fixed. Neither of them are sophisticated enough to find out why others are behaving in the way they do and to out-smart them. Note also that these agents do not try to copy the production level chosen by more profitable agents. Although there exists some reservation about the prevalence of such mimetic behavior in market set up based on experimental results (e.g, Bosch-Domènech and Vriend 2003), mimetic dynamics have been studied quite extensively in the literature. It would be an interesting future research to consider the interaction among our naive agents and such mimetic agents.

In particular, following the literature on agent-based model of learning in Cournot competition (Vriend 2000, Arifovic and Maschek 2006, Vallée and Yildizoğlu 2009), we apply the basic idea of the individual evolutionary learning (IEL) model (see Arifovic and Ledyard 2012 for a summary) in modeling how agents learn. IEL model is a convenient way to model adaptive learning when the number of strategies is very large. If there are only a few strategies, then we can use, for example, variants of the experienced weighted attraction (EWA) learning model (Camerer and Ho 1999) to implement similar learning processes. We assume that each agent is initially endowed with randomly selected $S$ strategies (quantities) $s \in[0, q M A X]$ where $q M A X=4.0 \times q^{W}(a, b, c, d, e, N)$. This formulation of upper bound is an arbitrary one. We have tested with other values. Similar results can be obtained as long as the upper bound changes appropriately (not too far away nor not too close from the equilibrium quantities) with the equilibrium quantities. Let $S_{0}^{i}$ be the initially generated strategy set for agent $i$. Each strategy (quantity) is represented by a binary string (chromosome) of length $L$. Therefore, there are $2^{L}$ possible strategies that equally divide the interval $[0, q M A X]$. For example, if $L=14$, strategy 00000000000000 represents quantity 0 and strategy 11111111111111 represents quantity $q M A X$. At any point in time, each agent has $S$ (not necessarily distinct) strategies out of these $2^{L}$ possible strategies in their strategy sets. In our version of IEL, learning takes place 


\section{Hanaki and Rouchier}

at two levels: within generation and across generations. Within a generation $g$, each agent $i$ learns which $s \in S_{g}^{i}$ to use in each period. We assume that one generation consists of $T$ periods. Across generations learning is represented by the evolution of $S_{g}^{i}$ from one generation to next by evolutionary selection. Thus, there are $T$ periods between two evolutionary selections. This was called "GA rate" by Vriend (2000). Below, we first describe the within generation learning and later across generations learning.

\subsubsection{Learning within a Generation}

Each period $t$, agent $i$ in generation $g$ whose strategy set is $S_{g}^{i}$ decides which $s \in S_{g}^{i}$ to choose according to the performance (attractiveness) of each strategy, $A_{s}^{i}(t, g)$. That is agent $i$ chooses its quantity (strategy) in period $t$ of generation $g, s^{i}(t, g)$, according to a probability

$$
\operatorname{Pr}\left(s^{i}(t, g)=s\right)=\frac{e^{\beta A_{s}^{i}(t, g)}}{\sum_{k \in S_{g}^{i}} e^{\beta A_{k}^{i}(t, g)}}
$$

$\beta$ in Equation (5) represents the sensitivity of within a generation choice to within generation performance of each strategy up to period $t$. When $\beta=0$, agent is choosing its quantity randomly from $S_{g}^{i}$ each period. And when $\beta \rightarrow \infty$, agent chooses the quantity that has resulted in the highest performance so far (from the beginning of the generation). This formulation using a logistic function is now commonly used in many behavioral models such as the EWA model (Camerer and Ho 1999), the logit equilibrium (Goeree and Holt 2001), and Quantal Response Equilibrium (McKelvey and Palfrey 1995).

The performance (attractiveness) of a strategy $s$ for agent $i$ in generation $g$ in period $t, A_{s}^{i}(t, g)$, evolves based on its past performance as follows:

$$
A_{s}^{i}(t+1, g)=A_{s}^{i}(t, g)+R_{s}^{i}(t, g)
$$

where $A_{s}^{i}(0, g)=0$ for all $i, g$, and $s \in S_{g}^{i}$ and

$$
R_{s}^{i}(t, g)= \begin{cases}\frac{\left(P^{i}(t, g)-s\right) s-C(s)}{T} & \text { if } \mathrm{i} \text { is an Ignorant } \\ \frac{\left(a-b s-c Q_{-i}(t, g)-s\right) s-C(s)}{T} & \text { if } \mathrm{i} \text { is an Informed }\end{cases}
$$

where $Q_{-i}(t, g)$ total quantity chosen by other agents, $P^{i}(t, g)$ is the realized price for agent $i$ (Equation $(1)), s^{i}(t, g)$ is the strategy chosen by agent $i$ in period $t$ of generation $g$, and $C(s)$ is cost of taking strategy $s$ (Equation (2)). As noted above $T$ is the length of one generation.

As one can see from the equations of $R_{s}^{i}(t, g)$ for Ignorant and Informed (Equation (7)), these two types of agents have different information and ideas regarding the functioning of the market. Ignorant agents assume the realized price in the previous period to be constant in evaluating potential profit they could have obtained if they have chosen other production levels (as can be seen by use of $p^{i}(t, g)$ in the equation). That is, ignorant agents don't consider the effect of their decisions on the market prices because they do not know and learn about the demand function they face. On the other hand, informed agents know the demand function they face, and take the effect of their decisions on the market prices into account. What these informed agents do not consider is the possibility of other agents changing their production levels (as can be seen by the use of $Q_{-i}(t, g)$ which takes the total production of others agents in the previous period given). It is clearly the case that neither type of agents is very sophisticated. Yet, between these two types, informed agents are more sophisticated than ignorant agents. We now turn to description of across generations learning based on an evolutionary selection.

\subsubsection{Evolutionary Selections}

As noted above, an evolutionary selection takes place every $T$ periods to modify the strategy set of each agent: $S_{g}^{i}$. For each agent $i$, we select $K$ strategies (out of $S$ ) by a tournament selection. The tournament 


\section{Hanaki and Rouchier}

Table 1: List of parameters and their values

\begin{tabular}{lc}
\hline Market and cost related parameters & \\
\hline Number of agents: $N$ & 20 \\
Number of Informed agents: $n_{f}$ & $0,1, \ldots, N\}$ \\
Intercept of inverse demand: $a$ & 200 \\
Own price effect: $b$ & 1.0 \\
Cross price effect: $c^{*}$ & $-0.1, \ldots, 1.0\}$ \\
Coefficients of cost 1: $d$ & 0.0 \\
Coefficients of cost 2: $e$ & 1.0 \\
\hline Parameters of IEL & 14 \\
Length of chromosome: $L$ & 100 \\
Number of strategy in each player's strategy set: $S$ & 0.01 \\
Intensity of choice parameter: $\beta$ & 100 \\
Length of one generation: $T$ & 3000 \\
Number of generation: & 60 \\
Preservation from previous generation strategies: $K$ & 0.2 \\
Intensity of selection in Tournament selection: $\tau$ & 0.001 \\
Mutation rate: $\mu$ & \\
\hline Note: $*$ Given other parameter values, the minimum value of possible $c$ is approximately -0.15789
\end{tabular}

takes place as follows: we randomly select (with replacement) $S \times \tau$ strategies, and choose the strategy with the highest $A_{s}^{i}(T, g)$ (shown in Equation (6)) among them. Here, $\tau \leq 1$ is called the selection pressure (Intensity of selection). We repeat this process for $K$ times to select $K$ strategies. These $K$ strategies will be kept as they are in the next generation strategy set, $S_{g+1}^{i}$. To introduce new $S-K$ strategies into $S_{g+1}^{i}$, we introduce crossover and mutation operation for $(S-K) / 2$ randomly chosen strategy pairs (from above $K$ ). Crossover operation based on a pair of randomly chosen strategy is to take the first $l$ bits of the chromosome from one of the strategy and the last $L-l$ bits of the chromosome from the other strategy and connect them to create a new pair of strategies. The exact position of $l$ is chosen randomly for every randomly chosen pair. Once these crossover operations generate $S-K$ strategies, the mutation operation, that is to flip each entry of the chromosome with probability $\mu$, will be carried out for these $S-K$ strategies.

\section{COMPUTATIONAL EXPERIMENTS}

We conduct a set of computational experiments by varying the number of ignorant $\left(n_{g}=N-n_{f}\right)$ and informed agents $\left(n_{f}\right)$, as well as the nature and the degree of strategic interactions among them, $c$, while fixing the values of other parameters. Table 1 summarizes the parameters of the model as well as their values used in the following computational experiments.

Given the value of parameter values shown in Table 1, the symmetric Cournot Equilibrium and the symmetric Walrasian Equilibrium quantities as functions of $c, q^{C}(c)$ and $q^{W}(c)$, are:

$$
\begin{aligned}
q^{C}(c) & =\frac{200}{4+19 c} \\
q^{W}(c) & =\frac{200}{3+19 c}
\end{aligned}
$$

\subsection{Results}

Our simulation consists of 3000 generations of 100 periods each. Below we report the results based on the last 600 generations where outcomes are stable. The speed of convergence, subjects to the random 


\section{Hanaki and Rouchier}

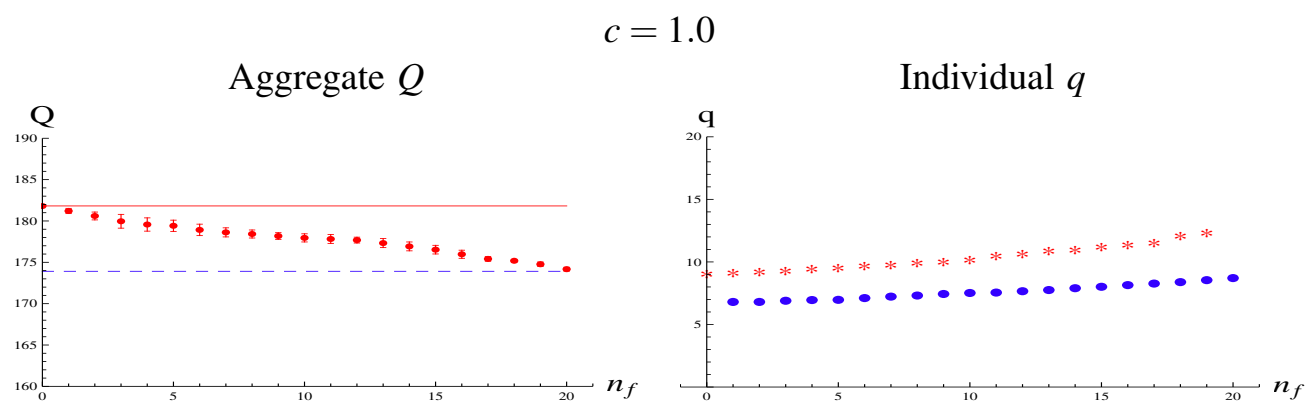

Figure 1: Mean total quantity $Q$ (left) and mean individual quantity $q$ (left) when goods are homogeneous $(c=1.0)$. Each point is based on averaging over 20 simulation runs using varying random seeds. For individual quantity, we take average across each type of agents: ignorant (red star) and informed (blue circles) in each simulation, and then average them over 20 simulation runs. The total quantities corresponding to symmetric Cournot equilibrium (blue, dashed), and Walrasian equilibrium (red, solid) are also shown.

introduction of strategies, differ depending on the value of $c$ as well as the composition of informed and ignorant agents. Thus for each agent, we take the average level of outputs (and profits) from these last 60000 periods of simulation.

\subsubsection{Homogeneous Good Case}

Figure 1 reports the outcomes of the simulations of the bench mark homogeneous good case $(c=1.0)$. The left panel shows the relationship between the number of informed ( $n_{f}$ on the x-axis) and the average total output per period (y-axis). The figure is generated by taking average across 20 simulation runs based on various random seeds. The outcomes under the symmetric Cournot-Nash equilibrium (blue-dashed) as well as the symmetric Walrasian equilibrium (red-solid) are also shown. These quantities are obtained by multiplying the symmetric equilibrium quantities derived in Equations (8) and (9) by the number of firms. On the right panel, the relationship between the number of informed and the average individual output per period for two types of agents (ignorant in red-stars and informed in blue-circles) are shown. As one can easily see, when all the agents are ignorant $\left(n_{f}=0\right)$, the average total output coincides with that under the symmetric Walrasian equilibrium. When all the agents are informed $\left(n_{f}=N=20\right)$, the average total output coincides with the one under the symmetric Cournot-Nash equilibrium. These results are consistent with previous studies, namely, Vriend (2000), Arifovic and Maschek (2006), Vallée and Yildizoğlu (2009). In between these two extremes, the more informed agents there are, the lower the total output is. And the total outputs always fall between two equilibrium levels.

Let's look at the relationship between the number of informed and individual output for two types. When two types co-exist, the informed agents always produce less than the ignorant agents. This is because while ignorant agents learn to produce the quantity that equates the marginal cost and the price, those informed learn to best respond against other agents. And due to the existence of the strategic substitution, informed agents best respond, by producing less, against ignorants who learn to produce more. This interaction can be seen in the left panel of Figure 2 which shows the average outputs for each type in one simulation run. Also shown in the figures are the average level of outputs that equate the price and the marginal cost for ignorant (red-line) as well as the average level of outputs that are best responses against all the others' outputs for informed (blue-line). As one can see, on average, ignorant agents learn to produce quantity that equate the price and the marginal cost while the informed learn to best respond.

A consequence of this interaction is that the average profits of ignorant agents become higher than those of informed agents when they co-exist because ignorant agents producing more than informed agents and all the agents face the same price, Of course, the larger the fraction of ignorant agents is, the lower the price becomes, and, therefore, the lower will be the profits for both type of agents. These can be seen 


\section{Hanaki and Rouchier}

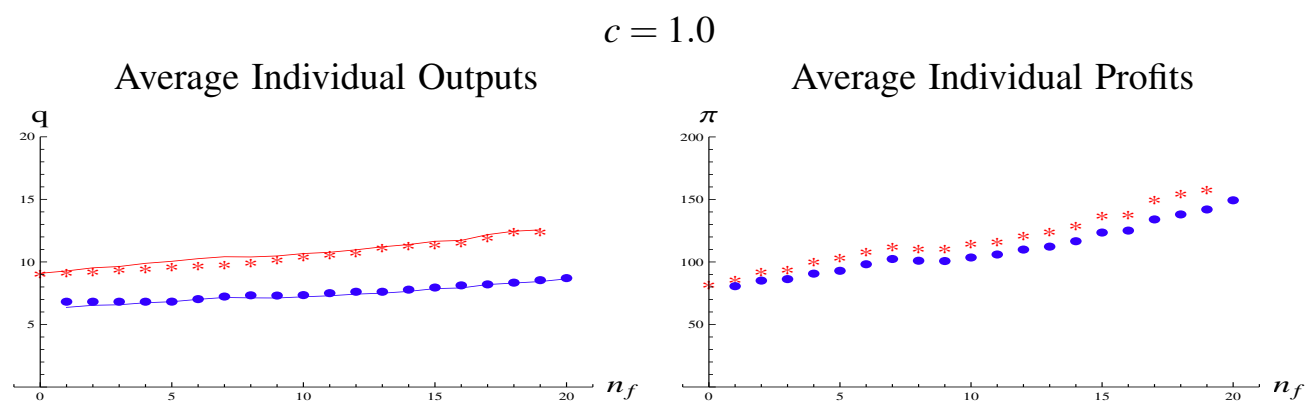

Figure 2: Average outputs (left) and profits (right) for each type. In the left panel, the average quantity that equate the price and the marginal cost for ignorant (red line) and the average quantity that are best response against all the others for informed (blue line) are shown. Result is based on one simulation run.

in the right panel of Figure 2. Thus, ignorant can obtain a higher profit than informed not by luck but because of the way they behave in this particular case.

\subsubsection{Differentiated Goods}

We have seen above that when the goods are homogeneous and agents compete in terms of quantity, ignorants can obtain higher profits than informed agents not by luck but because of the way they learned to behave. We have also seen above that the ignorant agents obtain a higher profit than informed agents because the formers learn to produce more while the latter learn to produce less. Does this result continue to hold when we move away from the homogeneous good oligopoly market and consider differentiated goods markets? A quick thought experiment tells us this will not be so.

Let's us imagine that all the agents are informed and that they learned to produce as in the Cournot-Nash equilibrium. Now, replace one of the informed with an ignorant. As before, the ignorant will soon learn to produce more. But it should be quite obvious from the inverse demand function that when goods are complement (thus, $c<0$ ), increasing one's quantity lowers own profit (by lowering the price one faces) but raises profits of others (because prices other agents face go up). Thus, ignorants who produce more than informed will not obtain higher profits when $c<0$. When goods are substitutes, on the other hand, an ignorant producing more does lower the profit of other agents as well. But whether it lowers the others' profits more than that of the ignorant's depends on the degree of substitution. To have such an effect, $c$ must be not be too low. Let's see these points by looking at our simulation results.

Figure 3 shows the results for $c=0.10$ (substitutes, top) and $c=-0.10$ (complements, bottom). For each value of $c$, we have three panels that show the relationship between number of informed $n_{f}$ and (a) the average per period total production $Q=\sum_{i} q_{i}$ (left), (b) the average per period outputs for ignorant (red stars) and informed (blue circles) (middle), and (c) the average per period profits for ignorant (red stars) and informed (blue circles) (right). The figures (including (c)) are all generated based on an average across 20 simulation runs with varying random seeds.

The close to linear relationships between the total outputs and the number of informed $n_{f}$ (shown in the left panel) are the same as what we have seen above for the $c=1.0$. It is also the case that the ignorants (shown in red) produce more than informed agents (shown in blue) when they co-exist (shown in the middle panel). But the the sign of the relationship between the number of informed and individual productions depends the nature of strategic interaction. Namely, when goods are substitutes $(c=0.10)$, the sign is positive, i.e., the higher the number of informed, the higher the average outputs for each type. On the contrary, when goods are complements (when $c=-0.10$ ), the sign is negative, i.e., the higher the number of informed, the lower the average outputs for each type. This difference is easy enough to understand. When goods are substitutes, others (in particular, ignorants) producing more reduces the price of one's good which makes an agent to produce less. Thus, the lower the number of ignorants is, the 


\section{Hanaki and Rouchier}
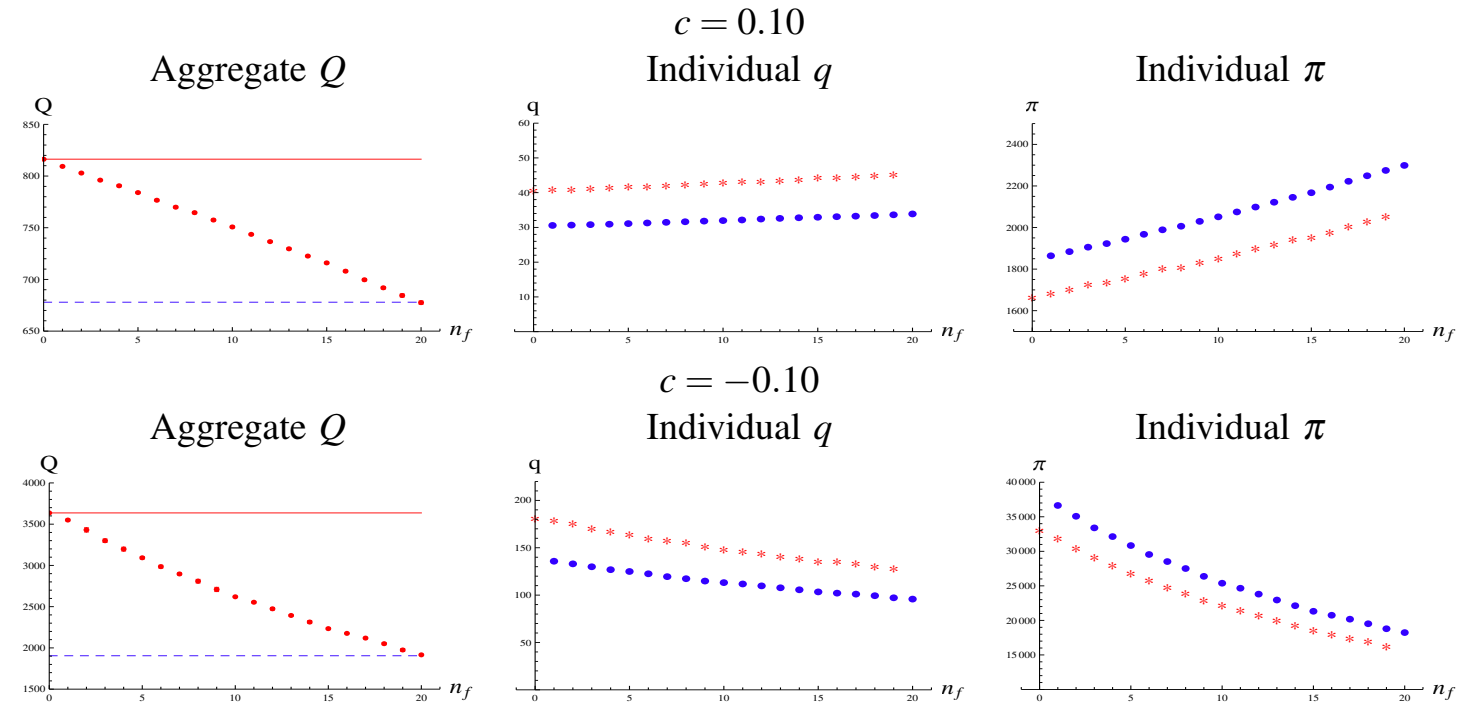

Figure 3: Mean total quantity $Q$ (left), mean individual quantity $q$ (middle), and mean individual profit $\pi$ (left). Each point is based on averaging over 20 simulation runs using varying random seeds. For individual quantity and profit, we take average across each type of agents (ignorants in red star and informed in blue circles) in each simulation, and then average them over 20 simulation runs. The total quantities corresponding to symmetric Cournot equilibrium (blue, dashed), and Walrasian equilibrium (red, solid) are also shown.

higher the individual production becomes. On the other hand, when goods are complements, others (again, in particular ignorants) producing more raises the price of one's good, which make an agent to produce more. Thus, the more ignorants there are, the higher the total production is. And, of course, this will cause higher order responses by other agents to increase their productions.

What should be noted in Figure 3 is individual profits shown in the right column. Both when $c=0.10$ and $c=-0.10$, the average profit of informed (blue circle) is higher than that of ignorant (red star). Thus, being ignorant does not offer any advantage in terms of profit when goods are weak substitutes or complements. It should also be noted that while the more informed agents there are, the higher the profits for both type in case of $c=0.10$, the opposite is true for $c=-0.10$. When goods are complements, the ignorant agents, who produces more than informed, increases everyone's profit.

Thus we have shown, based on simulations, that for ignorants to outperform informed agents in our differentiated oligopoly markets, goods have to be close substitutes. We have also shown, based on simulations, that ignorants learn to produce quantity that equate their marginal costs with the prices they face while the informed learn to best respond against others' production. By assuming symmetric behaviors within each type of agents, we can gain an analytical solution for our model, to which we turn next.

\subsection{A Mathematical Analysis}

When there are $n_{f}$ informed and $N-n_{f}$ ignorant agents, assuming the symmetry within each type, ignorant agents, who learn to equate the marginal cost (the right hand side of Equation (10)) and the price (the left hand side of Equation (10)) they face, will produce $q_{g}$ such that

$$
a-b q_{g}-c\left(n_{f} q_{f}+\left(N-n_{f}-1\right) q_{g}\right)=d+2 e q_{g}
$$

holds. Here $q_{f}$ is the quantity informed agents learn to produce. Therefore, we have

$$
q_{g}=\frac{a-d-c n_{f} q_{f}}{b+2 e+c\left(N-n_{f}-1\right)}
$$




\section{Hanaki and Rouchier}

Notice that when $n_{f}=0$, so that when all the agents are ignorants, this expression becomes the same as the symmetric Walrasian equilibrium quantity derived in Equation (4). Similarly, informed agents, again assuming the symmetry within each type, who learn to best respond against others, will learn to produce $q_{f}$ such that

$$
a-b q_{f}-c\left(\left(n_{f}-1\right) q_{f}+\left(N-n_{f}\right) q_{g}\right)-b q_{f}=d+2 e q_{f}
$$

hold. Equation (12) is simply the first order condition of profit maximization for the informed agent that equates the marginal revenue (the left hand side of Equation (12)) and the marginal cost (the right hand side of Equation (12)). Therefore, we have

$$
q_{f}=\frac{a-d-c\left(N-n_{f}\right) q_{g}}{2(b+e)+c\left(n_{f}-1\right)}
$$

Again, notice that when $n_{f}=N$, so that when all the agents are informed, this expression becomes the same as the symmetric Cournot-Nash equilibrium quantity derived in Equation (3).

Assuming the co-existence of informed and ignorant agents, and solving for $q_{g}$ and $q_{f}$ based on Equations (11) and (13), we have

$$
\begin{aligned}
q_{g}^{*} & =\frac{(a-d)(2(b+e)-c)}{Z} \\
q_{f}^{*} & =\frac{(a-d)(b+2 e-c)}{Z}
\end{aligned}
$$

where $Z=\left(b+2 e+c\left(N-n_{f}-1\right)\right)\left(2(b+e)+c\left(n_{f}-1\right)\right)-c^{2} n_{f}\left(N-n_{f}\right)$.

As one can easily see from Equations (14) and (15), $q_{g}^{*}>q_{f}^{*}$ as long as $b>0$ which is assumed in our model. We have observed $q_{g}^{*}>q_{f}^{*}$ in all of our simulations above. Now, we will consider the differences in the profit for ignorant, $\pi_{g}$, and informed, $\pi_{f}$, that is $\Delta \pi \equiv \pi_{g}-\pi_{f}$. Re-arranging the terms, we obtain

$$
\Delta \pi=(a-d)\left(q_{g}^{*}-q_{f}^{*}\right)-(b+e)\left(q_{g}^{* 2}-q_{f}^{* 2}\right)-c\left(n_{f}-\left(N-n_{f}\right)\right) q_{f}^{*} q_{g}^{*}-c\left(\left(N-n_{f}-1\right) q_{g}^{* 2}-\left(n_{f}-1\right) q_{f}^{* 2}\right)
$$

If we solve for $c \in(-(2 e+b) /(N-1), 1.0]$ such that $\Delta \pi=0$, we can obtain the critical value $c^{*}$ such that for $c>c^{*}$ that ignorant agents will obtain a higher profit than the informed agents. For the model we have considered, it is

$$
c^{*}=b+e-\sqrt{b e+e^{2}}
$$

which is independent of the fraction of informed in the population of the agents.

Figure 4 shows the relationship between $\Delta \pi$ and $c$ (based on Equation (16)) for three values of $n_{f}$ out of $N=20$ agents: 1 (red-solid), 10 (red-dashed), and 19 (blue-solid). We assume the same parameter values for demand and costs that we have used in our simulation analysis, that is, $a=200, b=1.0, d=0.0$, $e=1.0$. We only plot the relationship for $0.4 \leq c \leq 1.0$. What you can see is that for the three values of $n_{f}$ we have considered in the Figure $4, \Delta \pi=0$ at the same value of $c$ (approximately at $c=0.586$ ) as we have obtained in Equation (17) above.

\section{CONCLUSION}

In this short paper, we have considered interactions among two types of adaptive agents, ignorant and informed, in differentiated goods, as well as a homogeneous good, Cournot competition. We have assumed that agents learn how much to produce based on the past outcomes. Ignorant agents do not know about the demand function for their products and naively assume prices for their products will remain the same in the next period as the current period. Informed agents, on the other hand, know about the demand function, and learn how much to produce by myopically best responding against the quantities produced by others in the previous period, that is they consider the changes in prices of their products when they 


\section{Hanaki and Rouchier}

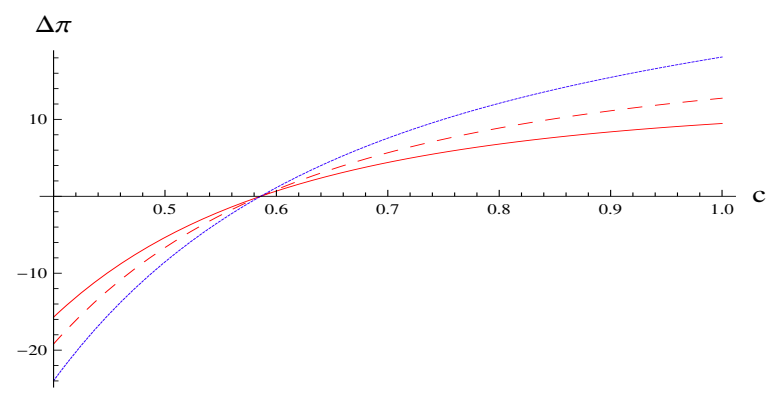

Figure 4: Relationship between $\Delta \pi$ and $c$ for the same parameter values that we have used in our simulation analysis. $N=20, a=200, b=1.0, d=0.0, e=1.0 . n_{f}=1$ (red, solid), $n_{f}=10$ (red, dashed), and $n_{f}=19$ (blue, solid).

change their quantities assuming the others keep their outputs fixed. We have also assumed that neither knows explicitly how others make their decisions (or learn). In this simple set-up, we can say that informed ones are smarter than ignorants, although even informed are naive as they are not aware (nor learn) about the heterogeneity among agents.

Within this simple set-up, we asked the following question: Can ignorant agents obtain higher profits than informed ones not by luck but due to the way they behave? We showed the answer is positive when goods are sufficiently substitutable to each others. But when goods are weak substitutes or complements, the informed will obtain higher profits than ignorants.

To include other types of adaptive agents into the model will be an interesting future research. For example, Anufriev, Kopányi, and Tuinstra (2012) consider interaction of OLS learning and gradient learning in a differentiated-goods Bertland (price) competition, and show that complex cyclical pricing patterns can emerge. Another aspect we have not considered is a possibility of agents changing their types. If we view various adaptive rules as merely various heuristics employed by agents, it is reasonable that agents switching from one heuristics to another depending on their performances. We leave introducing such switching possibilities into the analysis as future research as well.

\section{ACKNOWLEDGMENTS}

We thank Alan Kirman for suggesting this title. This project is partly supported by French-Japanese (ANR-JSPS, CHORUS) bilateral research project "BECOA" and French University Institute (L'Institut Universitaire de France).

\section{REFERENCES}

Anufriev, M., D. Kopányi, and J. Tuinstra. 2012, August. "Learning Cycles in Bertrand Competition with Differentiated Commodities and Competing Learning Rules". CenDEF working paper. No. 12-05.

Apesteguia, J., S. Huck, and J. Oechssler. 2007. "Imitation - theory and experimental evidence". Journal of Economic Theory 136:217-235.

Arifovic, J., and J. Ledyard. 2012. "Individual evolutionary learning with many agents". The Knowledge Engineering Review 27 (Special Issue 2): 239-254.

Arifovic, J., and M. K. Maschek. 2006. "Revisiting Individual Evolutionary Learning in the Cobweb Model - Al Illustration of the Virtual Spite-Effect". Computational Economics 28:333-354.

Bao, T., C. Hommes, J. Sonnemans, and J. Tuinstra. 2012. "Individual expectations, limited rationality and aggregate outcomes". Journal of Economic Dynamics and Control 36:1101-1120.

Bosch-Domènech, A., and N. J. Vriend. 2003. "Imitation of Successful Behaviour in Cournot Markets". Economic Journal 113:495-524. 


\section{Hanaki and Rouchier}

Camerer, C., and T.-H. Ho. 1999, July. "Experience-weighted Attraction Learning in Normal Form Games". Econometrica 67 (4): 827-874.

Fehr, E., and J.-R. Tyran. 2008. "Limited Rationality and Strategic Interaction: The Impact of the strategic environment on nominal inertia". Econometrica 76 (2): 353-394.

Goeree, J. K., and C. A. Holt. 2001. "The Little Treasures of Game Theory and Ten Intuitive Contradictions". American Economic Review 91:1402-1422.

Haltiwanger, J., and M. Waldman. 1985. "Rational Expectations and the Limits of Rationality: An Analysis of Heterogeneity". American Economics Review 75 (3): 326-340.

Haltiwanger, J., and M. Waldman. 1989. "Limited Rationality and Strategic Complements: The implications for macroeconomics". Quarterly Journal of Economics 104:463-484.

Heemeijer, P., C. Hommes, J. Sonnemans, and J. Tuinstra. 2009. "Price stability and volatility in markets with positive and negative expectations feedback: An experimental investigation". Journal of Economic Dynamics and Control 33:1052-1072.

Huck, S., H.-T. Normann, and J. Oechssler. 1999. "Learning in Cournot Oligopoly - An Experiment". Economic Journal 109 (454): C80-C95.

Huck, S., H.-T. Normann, and J. Oechssler. 2000. "Does information about competitors' actions increase or decrease competition in experimental oligopoly markets?". International Journal of Industrial Organization 18 (1): 39-57.

McKelvey, R. D., and T. R. Palfrey. 1995. "Quantal response equilibria for normal form games". Games and Economic Behavior 10:6-38.

Offerman, T., J. Potters, and J. Sonnemans. 2002. "Imitation and belief learning in an oligopoly experiment". Review of Economic Studies 69:973-997.

Sutan, A., and M. Willinger. 2009. "Guessing with negative feedback: An experiment". Journal of Economic Dynamics and Control 33:1123-1133.

Vallée, T., and M. Yildizoğlu. 2009. "Convergence in the finite Cournot oligopoly with social and individual learning". Journal of Economic Behavior and Organization 72:670-690.

Vega-Redondo, F. 1997. "The Evolution of Walrasian Behavior". Econometrica 65 (2): 375-384.

Vriend, N. J. 2000. "An illustration of the essential difference between individual and social learning, and its consequences for computational analyses". Journal of Economic Dynamics and Control 24:1-19.

\section{AUTHOR BIOGRAPHIES}

NOBUYUKI HANAKI is a Professor of Economics at the Faculty of Economics and Management at Aix-Marseille University (Aix Marseille School of Economics), and a member of GREQAM, CNRS. He is also a junior member of French University Institute. His research interest lies broadly in interactions among heterogeneous boundedly rational agents. He uses computational and laboratory experiments in addition to mathematical analyses in his research. He is currently an associate editor of Journal of Economic Behavior and Organization and Journal of Economic Interaction and Coordination. His email address is nobuyuki.hanaki@univ-amu.fr and his web page is http://www.vcharite.univ-mrs.fr $/ \sim$ nobi/index.html.

JULIETTE ROUCHIER is a Researcher at GREQAM, CNRS and a member of Aix-Marseille School of Economics. Her research interest lies in cognitive economics, with a special interest for the building of trust among interacting agents, the formation of prices in decentralized markets, and diffusion of opinions and beliefs. More recently she has turned to study the institutional setting that enable agents to interact so as to use a renewable resource. She employs multi-agent social simulations and field studies to inform her models. Her email address is juliette.rouchier@univ-amu.fr and her web page is http://www.vcharite.univ-mrs.fr/PP/rouchier/indexEnglish.html. 